\title{
BMJ open Science without meritocracy. Discrimination among European specialists in infectious diseases and clinical microbiology: a questionnaire
} survey

\author{
Evelina Tacconelli, ${ }^{1,2}$ Mario Poljak, ${ }^{3}$ Marina Cacace, ${ }^{4}$ Giovanni Caiati, ${ }^{4}$ \\ Nur Benzonana, ${ }^{5}$ Elisabeth Nagy, ${ }^{6}$ Titia Kortbeek ${ }^{7}$
}

To cite: Tacconelli E, Poljak M, Cacace M, et al. Science without meritocracy. Discrimination among European specialists in infectious diseases and clinical microbiology: a questionnaire survey. BMJ Open 2012;2:e001993. doi:10.1136/bmjopen-2012001993

- Prepublication history for this paper are available online. To view these files please visit the journal online (http://dx.doi.org/10.1136/ bmjopen-2012-001993).

Received 24 August 2012 Accepted 25 October 2012

This final article is available for use under the terms of the Creative Commons Attribution Non-Commercial 2.0 Licence; see http://bmjopen.bmj.com

For numbered affiliations see end of article

Correspondence to: Evelina Tacconelli; Evelina.Tacconelli@med. uni-tuebingen.de

\section{ABSTRACT}

Objective: In 2009, in a European survey, around a quarter of Europeans reported witnessing discrimination or harassment at their workplace. The parity committee from the European Society of Clinical Microbiology and Infectious Diseases (ESCMID) designed a questionnaire survey to investigate forms of discrimination with respect to country, gender and ethnicity among medical professionals in hospitals and universities carrying out activities in the clinical microbiology (CM) and infectious diseases (ID) fields.

Design: The survey consisted of 61 questions divided into five areas (sociodemographic, professional census and environment, leadership and generic) and ran anonymously for nearly 3 months on the ESCMID website.

Subjects: European specialists in CM/ID.

Results: Overall, we included 1274 professionals. The majority of respondents (68\%) stated that discrimination is present in medical science. A quarter of them reported personal experience with discrimination, mainly associated with gender and geographic region. Specialists from South-Western Europe experienced events at a much higher rate $(37 \%)$ than other European regions. The proportion of women among full professor was on average $46 \%$ in $\mathrm{CM}$ and $26 \%$ in ID. Participation in high-level decision-making committees was significantly ( $>10$ percentage points) different by gender and geographic origin. Yearly gross salary among CM/ID professionals was significantly different among European countries and by gender, within the same country. More than one-third of respondents (38\%) stated that international societies in $\mathrm{CM} / \mathrm{ID}$ have an imbalance as for committee member distribution and speakers at international conferences.

Conclusions: A quarter of CM/ID specialists experienced career and research discrimination in European hospitals and universities, mainly related to gender and geographic origin. Implementing proactive policies to tackle discrimination and improve representativeness and balance in career among CM/ID professionals in Europe is urgently needed.

\section{ARTICLE SUMMARY}

Article focus

- To investigate forms of discrimination with respect to country, gender and ethnicity among medical professionals in hospitals and universities carrying out activities in the clinical microbiology (CM) and infectious diseases (ID) fields.

Key messages

- Gender differences in career levels for CM/ID specialists, both academic and non-academic, are significant. A quarter of CM/ID specialists experienced career and research discrimination in European hospitals and universities, mainly related to gender and geographic origin. Perception of discrimination on the basis of the geographical region of work is more widespread among Eastern and Southern Eastern European countries.

- The burden of family care is disproportionally weighing on the shoulders of women. A huge gap divides men and women who are able to find a satisfactory balance between their work and family commitments.

- Implementing proactive policies to tackle discrimination and improve representativeness and balance in career among CM/ID professionals in Europe is urgently needed.

Strengths and limitations of this study

- First study performed at the European level specifically addressing the issue of discrimination in research and career among medical specialists.

- Survey questionnaire designed to target an extremely varied set of potential respondents, including people of different gender, age, ethnicity, nationality, religion, sexual orientation, disciplinary specialisation, professional role and level. 


\section{INTRODUCTION}

In 2000, the European Union adopted two very farreaching laws to prohibit discrimination in the workplace based on racial or ethnic origin, religion, disability or sexual orientation. ${ }^{1}$ In 2009, a survey was conducted to track, at the European level, perceptions and opinions on this field. ${ }^{2}$ Overall, around a quarter of Europeans reported witnessing discrimination or harassment and $16 \%$ of them experiencing it within 1 year of the study. Discrimination on ethnic origin $(61 \%)$ was perceived to be the most widespread grounds for discrimination, followed by discrimination based on age $(58 \%)$, disability (53\%) and gender $(40 \%){ }^{2}$

Discrimination in the academic area is more difficult to analyse although many of the factors making scientific work settings unfriendly to women and underrepresented minority groups are as common as in any other professional environment. ${ }^{3}{ }^{4}$ In particular, in medical professional settings, discrimination is harder to address because medical science itself (as science in general) is considered to be objective and an area where personal success is only based on scientific merit. ${ }^{2}$ The common belief of the blindness of science with respect to any personal feature of scientists is the basis of the subtle, informal and hardly visible nature of its segregating mechanisms, which often goes unrecognised even by those who are negatively affected by them. ${ }^{5}$ When it comes to scientific professions, such as the medical one, it is also paramount to identify which are the real positions where leadership is expressed. In fact, these professions have undergone deep transformation in the last few decades, with new roles and new professional figures emerging, modification of hierarchies and career paths and changes in the relative values attributed to different skills and capacities. ${ }^{6} 7$

Over the last few years, many projects have been focused on characterising discrimination at work level in academic settings. ${ }^{6-11}$ Most important factors reported to contribute to the so-called 'chilly climate' for women and minority groups in science are the following: exclusion from informal networks and the existence of 'hidden quotas' for women's and minority groups' presence in high-level positions, ${ }^{12}$ pay gap, ${ }^{6}$ access to resources for research and early-stage career development ${ }^{13}$ and evaluation of scientific merit. ${ }^{6}$ Gender discrimination, in particular, has been the focus of different European researches. ${ }^{6}{ }^{8-10} 14$ The 'She figures 2009' project, a collaboration between the Scientific Culture and Gender Issues Unit of the Directorate-General for Research of the EU Commission and the Helsinki Group, showed that although the feminisation of the student population is one of the most striking aspects of the evolution of research over the last 30 years, ${ }^{15}$ in most European countries women's academic career remains markedly characterised by strong vertical segregation. Women represented $44 \%$ among university students and $18 \%$ among full professors. Even of more interest, of all countries observed, there was none where female wages were equal to men's, despite the almost universal existence of legislation to impose gender wage equality. ${ }^{6}$

More difficult is to look for an evidence of discrimination related to ethnic origin or belonging to any other under-represented minority in medical academic area. Most observations derive from the US Universities where, in 2008, African-Americans, Hispanics and Native Americans made up more than a third of the US population but only $8.7 \%$ of physicians and $15 \%$ of enrolment in medical schools. ${ }^{16}$

In order to verify if, and to what extent, forms of discrimination exist, the Parity Commission of the European Society of Infectious Diseases and Clinical Microbiology (ESCMID) organised a pan-European questionnaire survey. This study was designed to explore different forms of discrimination with the main focus on gender, geographic origin and belonging to a minority among clinical microbiology (CM) and infectious diseases (ID) professionals working in European hospitals and universities.

\section{METHODS}

A literature review was performed in order to define evidence and causes for discrimination in medical science and among $\mathrm{CM} / \mathrm{ID}$ specialists. After assessing content validity, a pilot study including 10 participants from different European countries evaluated its acceptance and reproducibility, and results led to minor amendments to its format. The final survey (http://www.escmid.org/profession career/parity_commission/parity_survey/) consisted of 61 questions divided in five areas (socio-demographic, professional census and environment, leadership and generic) and ran anonymously for nearly 3 months (from 17 March 17 to 7 June 2011) on the ESCMID website (see table 1). All ESCMID regular members, attendees of the 21st European Congress of Clinical Microbiology and Infectious Diseases (ECCMID)/27th International Congress of Chemotherapy held in Milan, Italy and national/international ESCMID-affiliated societies' members were invited to participate.

\section{Sample size calculation}

Before administering the questionnaire a statistical description of the universe of reference (ie, European CM/ID specialists) according to geographic region and gender was accomplished based on information provided by the ESCMID. Since the type of sampling was of a nonprobabilistic nature, it was not possible to exactly determine the statistical representativeness of the sample. However, sample size to attain a confidence level of $95 \%$ and a precision of $\pm 5 \%$ was calculated as for a random sampling. ${ }^{17}$ A minimum of 400 respondents were set up to fulfil confidence level and precision. Using the simple random sampling we also calculated that with a sample overcoming 1000 units, with a confidence level of $95 \%$, the precision of the esteem could be fixed, by analogy, at $\pm 3 \%$. Poststratification operations were also accomplished 
Table 1 Questionnaire description

\begin{tabular}{|c|c|c|}
\hline Area & $\begin{array}{l}\text { Number of } \\
\text { questions }\end{array}$ & Description \\
\hline $\begin{array}{l}\text { Socio-demographic } \\
\text { Qs. 1-15 }\end{array}$ & 15 & $\begin{array}{l}\text { Qs. collecting basic information about demographic and sociographic } \\
\text { variables. }\end{array}$ \\
\hline $\begin{array}{l}\text { Professional census } \\
\text { Qs. 16-23 }\end{array}$ & 8 & $\begin{array}{l}\text { Qs. collecting information about specialty, academic and professional } \\
\text { achievement and employment status. }\end{array}$ \\
\hline $\begin{array}{l}\text { Professional environment } \\
\text { Qs. 24-35 }\end{array}$ & 12 & $\begin{array}{l}\text { Qs. collecting information on the respondent's experience/opinion about } \\
\text { discrimination regarding three features of the professional environment: } \\
\text { organisational cultures and behaviours; career support and work-life } \\
\text { balance. }\end{array}$ \\
\hline Leadership Qs. 36-56 & 21 & $\begin{array}{l}\text { Qs. collecting information on the respondent's experience/opinion about } \\
\text { discrimination regarding the attainment of leadership positions, } \\
\text { distinguishing between discrimination affecting research practice, } \\
\text { discrimination in the attainment of decision-making positions in research } \\
\text { management and discrimination in scientific communication. }\end{array}$ \\
\hline General Qs. 57-61 & 5 & Qs. collecting last impressions, comments and very general opinions. \\
\hline
\end{tabular}

and respondents were weighted according to gender and geographical region of work. We consider a significant result only those showing gaps as wide as, at least, 10 percentage points (corresponding to a precision of $\pm 5 \%$ ).

\section{Analysis of the main sources of discrimination}

Determinants of discrimination were grouped into three main areas: professional life (including career issues and working environmental issues), discrimination process and work-life balance. As for the sources of discrimination, results were systematically processed by gender and geographical region. The 40 European countries were classified into five geographical regions: Western Europe (WE), Northern Europe (NE), Eastern Europe (EE), South-WE (SWE) and South-EE (SEE), according to the standard ESCMID criteria (see online supplementary annex 1). To assess salary differences, each respondent was asked to declare his/her gross-income level in Euros compared to six predefined income-classes in the questionnaire: $<25000$ EUR; 25 000-44999 EUR; 45 000-64 999 EUR; 65 000-94 999 EUR; >125 000 EUR.
To simplify presentation of the survey's results, three indexes were built (see table 2): Professional Achievement Index (PAI), Work-Life Balance Index (WLBI) and Gender Discrimination Perception Index (GDPI). To define each index a 3-step procedure was applied: selecting and weighing indicators, assembling the index for each respondent and creating aggregates of respondents based on the index (low/medium/high).

\section{RESULTS}

Overall, 1566 individuals participated in the survey. Among them, 1274 working in European countries at the time of the study were included in the analysis. Respondents' distribution by gender, geographical region and age is shown in table 3 .

Table 4 illustrates the main professional achievement reached by 1274 respondents. Half of the interviewed individuals $(51 \%)$ reported to be involved in an academic career. Overall, a clear imbalance in the number of women in the highest level of academic career was

Table 2 Definition of indexes according to indicators

\begin{tabular}{|c|c|c|}
\hline Acronym & Index & Indicators \\
\hline PAI & Professional Achievement Index & $\begin{array}{l}\text { Peer-reviewed articles published in career } \\
\text { Funding received for research as principal investigator (2008/2010) } \\
\text { Membership in professional boards and committees }\end{array}$ \\
\hline WLBI & Work-Life Balance Index & $\begin{array}{l}\text { Responsibility for household duties } \\
\text { Having the number of children desired } \\
\text { Having/having not to discontinue career opportunities } \\
\text { Caring for an elderly or an ill parent or relative }\end{array}$ \\
\hline GDPI & $\begin{array}{l}\text { Gender Discrimination Perception } \\
\text { Index }\end{array}$ & $\begin{array}{l}\text { Reporting gender discrimination events } \\
\text { Perception of gender inequality in international societies } \\
\text { Perception of low representativeness, as for gender, of speakers at } \\
\text { international conferences } \\
\text { Belief that gender impacts on career's opportunities }\end{array}$ \\
\hline
\end{tabular}


Table 3 Main epidemiological characteristics of 1274 participants, by gender

\begin{tabular}{|c|c|c|c|c|c|c|}
\hline & Females $\mathrm{N}=784$ & $\%$ & Males $\mathrm{N}=490$ & $\%$ & Total & $\%$ \\
\hline Region* Western Europe & 244 & 31.1 & 195 & 39.8 & 439 & 35.5 \\
\hline Northern Europe & 96 & 12.2 & 61 & 12.4 & 157 & 12.3 \\
\hline Eastern Europe & 127 & 16.2 & 49 & 10.0 & 176 & 13.8 \\
\hline South-Western Europe & 137 & 17.5 & 79 & 16.2 & 216 & 17.0 \\
\hline South-Eastern Europe & 180 & 23.0 & 106 & 21.6 & 286 & 22.4 \\
\hline Year of age $<35$ & 251 & 32.0 & 102 & 20.8 & 353 & 27.7 \\
\hline $35-49$ & 331 & 42.2 & 217 & 44.3 & 548 & 43.0 \\
\hline $50-64$ & 189 & 24.1 & 155 & 31.6 & 344 & 27.0 \\
\hline$>65$ & 13 & 1.7 & 16 & 3.3 & 29 & 2.3 \\
\hline Specialty clinical microbiology & 384 & 48.9 & 216 & 44.1 & 600 & 47.0 \\
\hline Infectious diseases & 263 & 30.9 & 227 & 46.3 & 539 & 42.3 \\
\hline
\end{tabular}

detected. Women accounted on average for $36 \%$ of full professorship in CM/ID. However, the difference is significantly more evident for ID than CM professionals (26\% vs $46 \%$; see figures 1 and 2). Women are even more under-represented in boards and committees with more than 20 percentage points dividing males from females (see table 4). The most striking difference was observed for participation in editorial boards (34\% of females vs $66 \%$ of men). The presence in high-level decision-making bodies is also strongly influenced by geographic regions. Highest participation in expert panels is observed in professionals from NE (45\%) and lowest in those from EE (26\%; see online supplementary annex 2).

The number of publications in peer-reviewed journals, considered as the most traditional measure of scientific authority, shows clear gender differences (see table 4) and less evident regional differences (data not shown).
Interestingly, a similar percentage of men and women report their name not being mentioned as first author when they were the ones conceiving the scientific publication and making the greater contribution to it $(41 \%$ of men vs $37 \%$ of women). Age discrimination stands out as the most recurrent explanation given by $22 \%$ of respondents.

Yearly gross salary among ICM/ID professionals is significantly different among European countries. The highest ( $>10$ percentage points) salary gap is observed among women from EE and SEE. After selecting highincome level only ( $>95000 \mathrm{EUR} /$ year), the percentage of inclusions ranges from 5\% among professional females from EE to $42 \%$ of males in WE (see figure 3 ).

The majority of respondents $(68 \%)$ agreed that discrimination exists in scientific fields such as the medical science (see table 5). Respondents from SWE show the strongest commitment to gender issues while those from

Table 4 Main professional achievement of respondents, by gender

\begin{tabular}{|c|c|c|c|c|c|}
\hline & Females & $\%$ & Males & $\%$ & Total \\
\hline Professional level $(\mathrm{N}=1008)$ Head of division & 82 & 37.1 & 139 & $62.9^{\star}$ & 221 \\
\hline Head of ward & 109 & 48.2 & 117 & 51.8 & 226 \\
\hline Consultant & 156 & 44.3 & 196 & 55.7 & 352 \\
\hline University career $(\mathrm{N}=649)$ Full professors & 37 & 36.3 & 65 & $63.7^{\star}$ & 102 \\
\hline Associate professors & 48 & 34.8 & 90 & $65.2^{*}$ & 138 \\
\hline Assistant professors & 59 & 52.7 & 53 & 47.3 & 112 \\
\hline Lecturers & 72 & 47.7 & 79 & 52.3 & 151 \\
\hline Memberships $(\mathrm{N}=1274)$ Expert panels & 169 & 35.0 & 314 & $65.0^{\star}$ & 483 \\
\hline Project evaluation committees & 164 & 38.6 & 261 & $61.4^{\star}$ & 425 \\
\hline Program committees & 133 & 37.7 & 220 & $62.3^{\star}$ & 353 \\
\hline Advisory boards & 122 & 34.1 & 236 & $65.9^{\star}$ & 358 \\
\hline Recruitment committees & 106 & 37.9 & 174 & $62.1^{*}$ & 280 \\
\hline Editorial boards & 100 & 34.0 & 194 & $66.0^{\star}$ & 294 \\
\hline Publications in peer-reviewed journals $(N=1188)>200$ & 4 & 14.3 & 24 & $85.7^{\star}$ & 28 \\
\hline $101-200$ & 25 & 33.3 & 50 & $66.7^{\star}$ & 75 \\
\hline $51-100$ & 43 & 36.1 & 76 & $63.9^{\star}$ & 119 \\
\hline $11-50$ & 160 & 42.9 & 213 & 57.1 & 373 \\
\hline$<=10$ & 356 & $60.0^{*}$ & 237 & 40.0 & 593 \\
\hline
\end{tabular}


Figure 1 Proportion of 214 clinical microbiology professionals in different academic grades stratified by gender. †Significant results: 10 percentage points difference (corresponding to a precision of $\pm 5 \%$ ); prof:: professor.

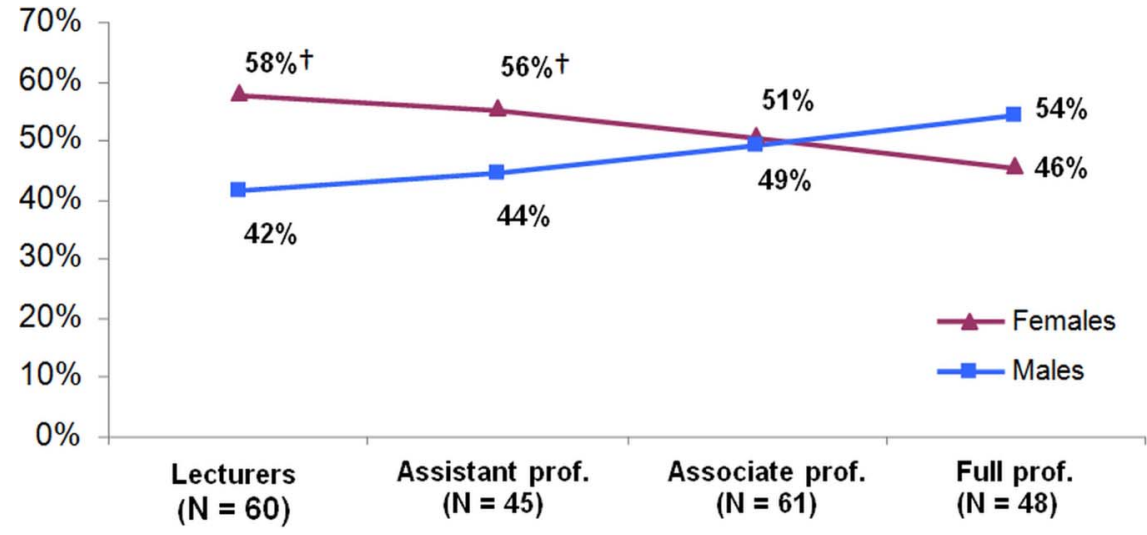

WE and NE are in an intermediate position and those from EE and SEE less agree on the presence of discrimination among ID/CM specialists (see table 6). A quarter of respondents $(26 \%)$ reported witnessing or experiencing discrimination or harassment at their workplace (37\% among women and $16 \%$ among men). As for regional differences, respondents from SWE countries report discrimination events at a much higher rate $(37 \%)$ than average $(26 \%)$, with the largest difference for gender while discrimination on the grounds of religion is more frequently denunciated from SEE countries $(8 \%$; see table 5$)$.

More than one-third (37\%) of respondents believe that $\mathrm{CM} / \mathrm{ID}$ international societies have discrimination problems, mainly related to country misbalance (28\%) and gender inequality (15\%). More than $20 \%$ believe that the most important international $\mathrm{CM} / \mathrm{ID}$ conferences (ECCMID and Interscience Conference on Antimicrobial Agents and Chemotherapy) are not balanced mainly for a speaker's geographic region of origin $(57 \%)$. The majority of respondents $(72 \%)$ believe that international societies should play a major role in helping universities and hospitals in addressing careerrelated issues and related discriminatory events (box 1).

The number of children reported might be considered as an indirect hint of the greater difficulty of women in CM/ID fields in reconciling work and family life. More than 20 percentage points divided the number of women and men who have two or more children (35\% of women vs $56 \%$ of men). Analysis of geographical distribution shows that there are relevant differences among European regions having two or and SE $38 \%$. Considering different age classes, people under 35 years strongly regret having to wait longer than desired for having a child. People in the age class 35-49 years, on the other hand, more often report having less children than desired, or not at all, because of work commitments ( $36 \%$ of women and $19 \%$ of men).

As expected, household duties are strongly influenced by gender. Women from SWE largely outnumber men as the ones who take up the greater part $(75 \%)$ of household duties: $68 \%$ of women vs $7 \%$ of men. After stratifying for age, while women under age 35 performing $100 \%$ of domestic chores are $29 \%$ and then the percentage slight decreases over the years, the trend for males is completely different. They perform all domestic duties until they presumably live alone (29\% under age 35), then the figure suddenly drops by 20 percentage points and remains very low for all other age classes.

The distribution of indexes summarises the major area of discrimination. The PAI distribution adjusted by age is shown in figure 4 . More than 20 points divide high professional achievements according to gender $(46 \%$ of men vs $26 \%$ of women) while regional differences are minimal (data not shown). The WLBI confirms the same distribution (see figure 5) with more than 30 percentage points of difference $(81 \%$ of men vs $52 \%$ of more children: NE 69\%, WE 47\%, SWE 40\%, EE $39 \%$
Figure 2 Proportion of 229 infectious diseases professionals in different academic grades stratified by gender. †Significant results: 10 percentage points difference (corresponding to a precision of $\pm 5 \%$ ); prof.: professor.

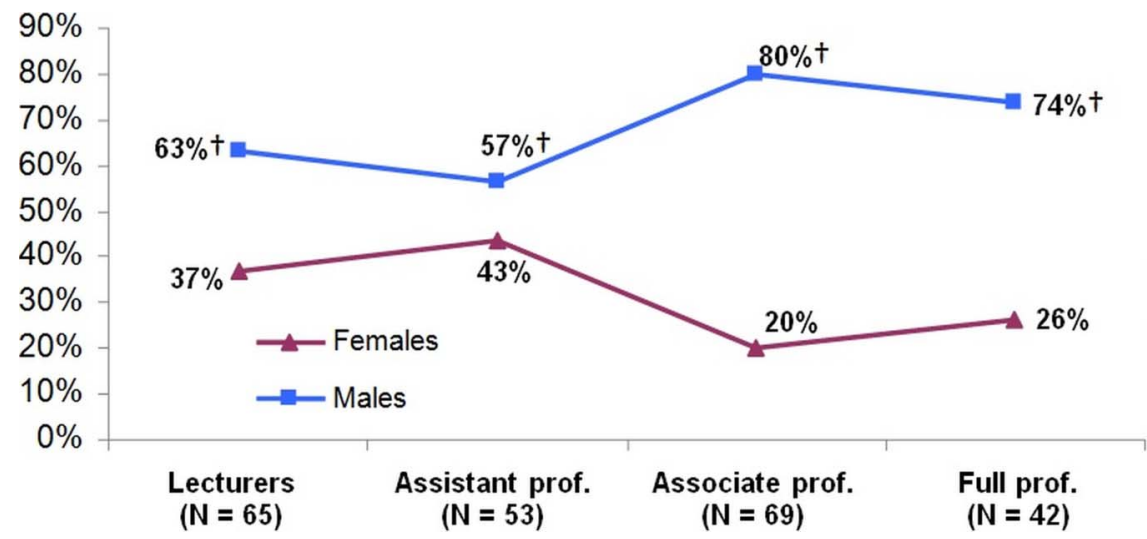




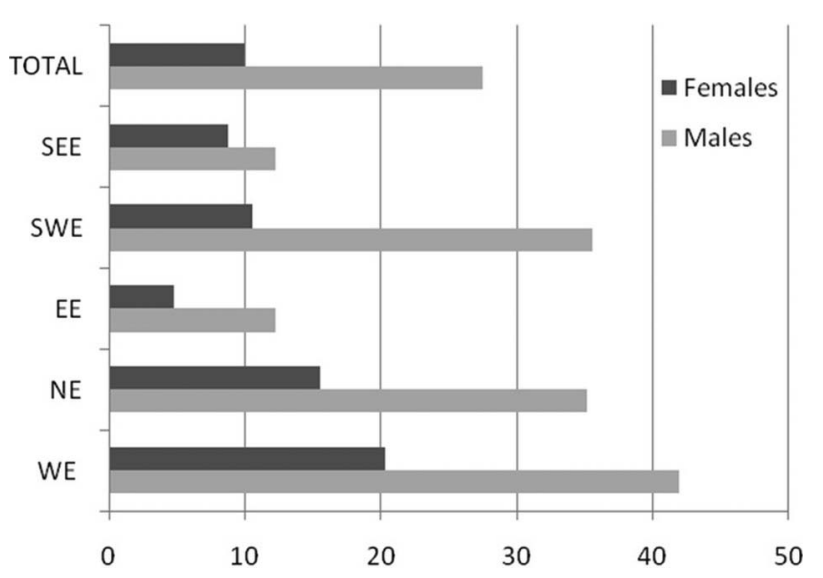

Figure 3 Proportion of 235 clinical microbiology/infectious diseases professionals in top income levels (>95 000 euro/ year) stratified by gender and geographic region.

women). Perception of gender discrimination according to the GDPI is presented in figure 6 with a significant difference between females and males $(26 \%$ vs $5 \%)$.

\section{DISCUSSION}

Three main conclusions can be drawn from our survey. First, gender differences in career levels for CM/ID specialists, both academic and non-academical, are significant. If gender differences in achievement are measured through substantial indicators of scientific and professional success, such as scientific publications, research funding and participation in boards and committees, large gaps are still recorded between men and women and are consistent among professionals working in all European regions. Second, more than two-thirds of $\mathrm{CM} / \mathrm{ID}$ professionals recognise the existence of discriminatory problems at their workplace. The perception of discrimination on the basis of the geographical region of work is more widespread among EE and SEE countries. Third, the burden of family care is disproportionally weighing on the shoulders of women. A huge gap divides men and women who are able to find a satisfactory balance between their work and family commitments. Even though there are important differences in women's situation in the different regions, the gap with men is always much wider in all regions, including NE countries (box 2).

Our data clearly show the gap in professorship's distribution between men and women. The data resemble the 'scissor phenomenon', a graphical representation of the divergence of men and women as they adopt senior positions in academic research, associate professorships and full professorships over time. Previous studies showed that although the number of women entering the first grade of medical school outnumbered that one of men, they then suddenly, after $\mathrm{PhD}$, are no longer found in positions of relevance in any universities across Europe. ${ }^{6}{ }^{10}$ Among CM and ID professionals the difference in different academic grades stratified by gender is significantly more evident for ID than CM professionals (26\% vs $46 \%)$. Wide differences were also observed according to geographic regions. There are many cultural, economic and organisational reasons behind the wide differences that do emerge for academic career among CM/ID specialists. It is worth noticing that the regions where the gender gap is smaller among full professors (NE, EE and SEE) are those where women outnumber men to a larger extent among CM professionals. In this field women enjoy a relatively less discriminating environment. However, it is important to underline that in many European countries, especially where microbiology is practising mainly as laboratory discipline and not as CM, the career path is less 'appealing' than other medical disciplines. This is not, however, the only variable having an impact, since situations are extremely varied. In daily 'real' university life we frequently hear that plausible 'explanation' from women not being represented at the same level of men at highest academic career is that women are less interested in academic career since this is not feasible with family and in particular with children care. The fact that women publish less than men is usually used as a key supporting argument. However, previous studies

\begin{tabular}{|c|c|c|c|c|c|c|}
\hline & Females & $\% *$ & Males & $\% *$ & Total & $\% *$ \\
\hline Existence of discrimination† & 461 & 73.8 & 363 & 57.5 & 824 & 68 \\
\hline At least one report & 230 & $36.8 \ddagger$ & 101 & 16.1 & 331 & 26.5 \\
\hline 1 Report & 175 & 28.0 & 75 & 12.0 & 250 & 20.1 \\
\hline 2 Reports & 34 & 5.4 & 14 & 2.2 & 48 & 3.8 \\
\hline$>2$ Reports & 21 & 3.4 & 12 & 1.9 & 33 & 2.6 \\
\hline Gender & 186 & $29.8 \ddagger$ & 39 & 6.2 & 225 & 18.0 \\
\hline Ethnicity & 32 & 5.1 & 20 & 3.2 & 52 & 4.2 \\
\hline Nationality & 57 & 9.1 & 43 & 6.9 & 100 & 8.0 \\
\hline Religious background & 27 & 4.3 & 19 & 3.0 & 46 & 3.7 \\
\hline Sexual orientation & 19 & 3.0 & 21 & 3.4 & 40 & 3.2 \\
\hline
\end{tabular}


Table 6 Proportion of CM/ID professionals declaring to have experienced or witnessed discrimination, by geographic region

\begin{tabular}{|c|c|c|c|c|c|c|}
\hline & WE N=578\% & NE $N=110 \%$ & EE $N=135 \%$ & SWE N=255\% & SEE $N=173 \%$ & Total $\mathrm{N}=1251 \%$ \\
\hline At least one rep. & 27.3 & $14.5 \dagger$ & 17.8 & $37.1 \dagger$ & 21.4 & 26.5 \\
\hline 1 Report & 21.6 & 10.0 & 15.6 & 27.2 & 15.1 & 20.1 \\
\hline 2 Reports & 3.5 & 1.8 & 1.5 & 5.5 & 5.2 & 3.8 \\
\hline$>\mathrm{R}$ reports & 2.2 & 2.7 & 0.7 & 4.7 & 1.7 & 2.6 \\
\hline Gender & 17.5 & 12.8 & 12.2 & $29.6^{*}$ & 10.1 & 18.0 \\
\hline Ethnicity & 4.2 & 2.1 & 1.2 & 6.6 & 4.1 & 4.2 \\
\hline Nationality & 7.8 & 4.9 & 5.5 & 11.2 & 8.1 & 8.0 \\
\hline Religious background & 3.9 & 1.1 & 0.5 & 3.0 & 8.1 & 3.7 \\
\hline Sexual orientation & 3.1 & 1.1 & 1.0 & 6.2 & 1.9 & 3.2 \\
\hline
\end{tabular}

*Significant results: 10 percentage points difference (corresponding to a precision of $\pm 5 \%$ ); $N=$ number.

CM, clinical microbiology; EE, Eastern Europe; ID, infectious diseases; NE, Northern Europe; SEE, South-EE; SWE, South-WE; WE, Western Europe.

documented that productivity is affected by a number of factors which are unrelated to scientific merit. ${ }^{15-21}$ Regional differences in publishing also suggest the existence of many determinants of scientific productivity. Professionals from WE and SWE are those with the highest rate of publications among CM/ID professionals. Creamer suggested that prolific publishers are disproportionately white males because the career paths, work assignments, research interests and access to resources conducive to frequent publishing are more characteristic of white men than of women and minorities. ${ }^{18}$ However, family responsibilities probably have less effect on women's publishing activity than work assignments and time. Finally, women's lower publishing rates may be a consequence of a 'chilly climate' in some academic departments. Women are more likely than men to be excluded and isolated from the types of professional and social networks that define the life of a department. As our survey shows, women and professionals from EE are less likely than men to receive visiting appointments and

\section{Box 1 Definitions of terms used in the article}

Meritocracy. It is a system of government or other administration (such as business administration) wherein appointments and responsibilities are objectively assigned to individuals based upon their 'merits', namely intelligence, credentials and education, determined through evaluations or examinations.

Discrimination: The treatment or consideration of, or making a distinction in favour of or against, a person based on the group, class or category to which that person belongs rather than on individual merit. It involves intentional behaviours towards such groups, for instance, formally or informally excluding or restricting members of one group from opportunities that are available to another group.

Inequality. The observable and measurable effects of discriminatory practices and habits, resulting in differential conditions of professionals within scientific and managerial careers.

Minorities: Minorities among healthcare workers were defined as those discriminated in their professional career and/or underrepresented in scientific societies because of gender, age, sexual orientation, racial, regional, religious and/or political reasons without consideration of their personal achievements. participate in high-level decision-making boards, activity that encourages the building of professional networks and contacts outside home institutions. In this sense, productivity appears as a function of one's position in the communication system in a discipline rather than personal scientific merits.

The analysis of gap salary related to gender and geographic regions is more difficult to analyse. A major limitation we encountered is related to the impossibility to adjust our results to national gross domestic product per capita corrected by local life costs. However, the pay gap between women and men is consistent within the same country in all European nations. Therefore, a need to implement proactive policies to tackle gender pay gap at the European level is crucial.

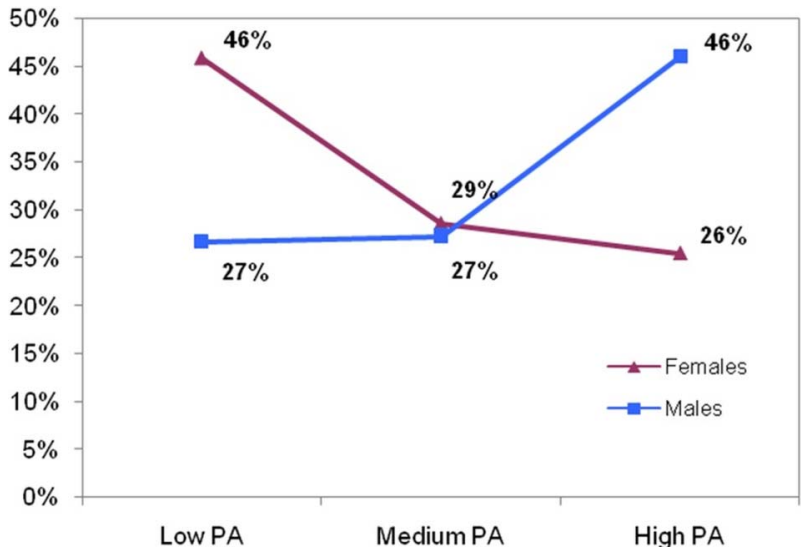

Figure 4 Professional achievements according to PAI stratified by gender. PAI: Professional Achievement Index. Each indicator was evaluated on a trichotomous ordinal scale (low/medium/high presence). Each mode was attributed a numerical value between 0 and 1 . The results obtained for each individual indicator were then added. To make the results of the different indexes homogeneous, the value scale of each index has been equalised at a 0-10 range. For each index a threshold system has been set up, so to place each individual within a 'low/medium/high' trichotomous scale with respect to a given profile (for instance: 'medium professional achievement'). See table 2 for indicators included in this index. 


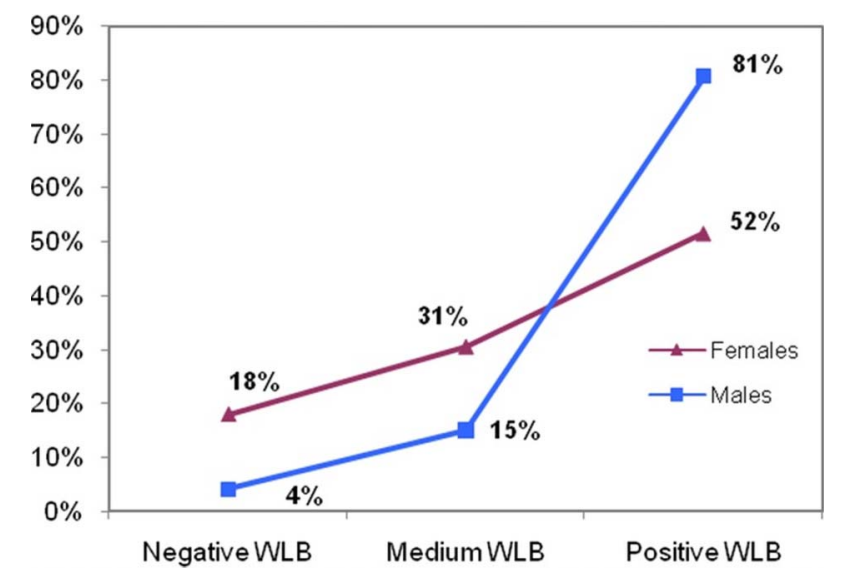

Figure 5 Work-life balance according to WLBI stratified by gender. WLBI: Work-Life Balance Index. Each indicator was evaluated on a trichotomous ordinal scale (low/medium/high presence). Each mode was attributed a numerical value between 0 and 1 . The results obtained for each individual indicator were then added. To make the results of the different indexes homogeneous, the value scale of each index has been equalised at a 0-10 range. For each index a threshold system has been set up, so to place each individual within a 'low/medium/high' trichotomous scale with respect to a given profile (for instance: 'positive work-life balance'). See table 2 for indicators included in this index.

Our data show that the issue of inequality in medical science is strongly perceived by the majority of $\mathrm{CM} / \mathrm{ID}$ professionals $(68 \%)$. Differences can be recorded across geographical areas, although there is no region where

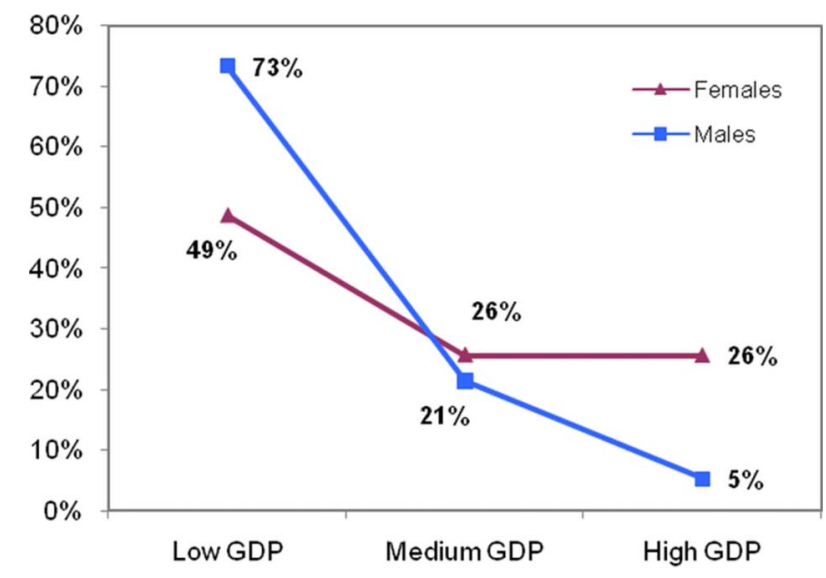

Figure 6 Perception of gender discrimination according to GDPI stratified by gender. GPDI: Gender Discrimination Perception Index. Each indicator was evaluated on a trichotomous ordinal scale (low/medium/high presence). Each mode was attributed a numerical value between 0 and 1. The results obtained for each individual indicator were then added. To make the results of the different indexes homogeneous, the value scale of each index has been equalised at a 0-10 range. For each index a threshold system has been set up, so to place each individual within a 'low/ medium/high' trichotomous scale with respect to a given profile (for instance: 'low perception of gender discrimination'). See table 2 for indicators included in this index.

\section{Box 2 Why is discrimination so hard to acknowledge}

- Difficult to recognise, since it is subtle, informal and generally embodied in well-established cultural, organisational and behavioural patterns.

- Annoying to admit, since it jeopardises one's opinion about the fairness and quality of the environment s/he works in, which may be experienced by some as an aggression to their professional identity.

- Somewhat depressing to admit, since it affects the selfesteem, self confidence and hopes of the potential victims of.

- Often counterproductive to admit, since it puts the hard-won credibility of women and under-represented groups at risk in the working environment.

consensus on the existence of discrimination falls below $66 \%$ of respondents, while if we only include those reporting substantial discrimination, we never fall below $50 \%$. Even of more interest a quarter of respondents reported witnessing or experiencing discrimination at their workplace mainly related to gender and nationality. The analysis of the work-life balance also showed significant differences according to gender. However, it is noteworthy that a substantial part of respondents, regardless of the gender, regret having to wait longer than desired for having a child or having less children than desired, or not at all, because of work commitments.

Finally, the use of indexes that, including different questions' results, was introduced to summarise and simplify main conclusions, further underlines that there is a dramatic difference among CM/ID specialists by gender regarding professional achievement, life satisfaction and perception of discrimination.

\section{Strengths and weakness of study}

This is the first study performed at the European level specifically addressing the issue of discrimination in research and career among medical specialists. Previous researchers analysed the issue mainly related to the scientific academic area and never specifically on the medical one. Since the importance of the researchers' selection and career development in medicine and the consequential impact on European citizens' health we do believe the definition of such a high level of perceived and actual career discrimination should be carefully evaluated by policymakers. Our study has many limitations. First of all, the ESCMID survey questionnaire was designed to target an extremely varied set of potential respondents, including people of different gender, age, ethnicity, nationality, religion, sexual orientation, disciplinary specialisation, professional role and level. It was obviously impossible, also considering it had to take the shortest time possible to be filled out, to adequately cover all aspects which would have deserved to be analysed if each of those qualities had to be taken in full consideration. It was also impossible to proceed with a reliable stratification 
according to more variables than gender and the geographic region of current employment.

\section{FURTHER RESEARCH}

Future researches should focus on some of the neglected issues, for instance targeting ethnicity, religion and sexual orientation in more details. To obtain a more accurate understanding of the $\mathrm{CM} / \mathrm{ID}$ reality as concerns equality issues other methodological approaches and sources of information should be added, such as direct observation, focus groups and qualitative interviews. It is also important to underline the tendency of professionals (both genders) to see scientific societies more involved than their own institutes in coping with parity issues. In particular, respondents seem to particularly value the role of international societies could play, beyond scientific exchange, as professional networks with a major role in dealing with professional issues, including those related to the work environment, work-life balance and career. Specific studies on international medical societies and parity issues should be therefore promoted in order to better understand these dynamics and to identify the real demands professionals express to societies and to institutes when parity issues are at stakes.

\section{Implications for clinicians and policymakers}

We believe that the results of our survey will play a major role in stimulating $\mathrm{CM}$ and ID specialists, other international societies for different specialisations as well as media to press policymakers to evaluate the fairness of academic career at the national level. However, we are aware that a significant improvement in the current situation at hospital and university level for medical specialists can be reached only through more direct involvement of policymakers at the European level. Informal exclusion ('hidden quotas') of new and young scientists just for being women or coming from a discriminated geographic region will definitively limit research and therefore will impact on future health of the Europeans citizens. In many countries, laws to define recruitment in the academic area, ensuring equal opportunities for all scientists, regardless of gender and country of origin, need to be introduced. Our final common goal should be to move from science without meritocracy towards science only based on meritocracy in a very near future.

\section{Author affiliations}

${ }^{1}$ Department of Infectious Diseases, Università Cattolica Sacro Cuore, Rome, Italy

2Division of Infectious Diseases, Dept. of Internal Medicine I,

Universitätsklinikum Tübingen, Germany

${ }^{3}$ Institute of Microbiology and Immunology, University of Ljubljana, Ljubljana, Slovenia

${ }^{4}$ ASDO, Women Assembly for Combating against Social Exclusion, Rome, Italy

${ }^{5}$ Department of Infectious Diseases and Clinical Microbiology, Dr. Lutfi Kirdar Kartal Education and Research Hospital, Istanbul, Turkey

${ }^{6}$ Institute of Clinical Microbiology, University of Szeged, Szeged, Hungary

${ }^{7}$ Centre for Infectious Disease Control Netherlands, Laboratory for Infectious
Diseases and Perinatal Screening, National Institute of Public Health and the Environment, Bilthoven, The Netherlands.

Acknowledgements The authors would like to express their gratitude to Karin Werner and Judith Zimmerman (ESCMID administrative office) in providing additional data and their support of this work.

Contributors ET had the idea of the research, contributed to the analysis and interpretation of the data, and drafted the paper. MC and GC contributed to the analysis and interpretation of the data. MP, NB, EN and TK contributed to the conception of the study, interpretation of the data and critically revised the manuscript.

Funding ESCMID provided a grant for the statistical assistance.

Competing interests None.

Provenance and peer review Not commissioned; externally peer reviewed

Data sharing statement Further summary data are available from the corresponding author at Evelina.Tacconelli@med.uni-tuebingen.de.

\section{REFERENCES}

1. Directive $2000 / 43 / E C$ of 29 June 2000 and Directive $2000 / 78 / E C$ of 27 November 2000.

2. Discrimination in the EU in 2009. Fieldwork May-June 2009. Special Eurobarometer 317.

3. European Commission 2000. Science Policies in the European Union: Promoting Excellence through Mainstreaming Gender Equality. ETAN Report, Luxembourg.

4. Santamaría A, Merino A, Viñas O, et al. Does medicine still show an unresolved discrimination against women? Experience in two European university hospitals. J Med Ethics 2009;35:104-6.

5. Keller EF. Reflections on gender and science. New Haven: Yale University Press, 1998.

6. European Commission 2009, She Figures 2009. Statistics and Indicators on Gender Equality in Science, Brussels.

7. Palomba R. Does gender matter in scientific leadership? In: Brouns M, Addis E, eds. OECD, Women in scientific careers. Unleashing the potential. Paris: OECD Publishing, 2006:121-5.

8. European Commission 2004. Gender and Excellence in the Making. Luxembourg.

9. European Commission 2007. Remuneration of Researchers in the Public and Private Sectors. Final report. Brussels.

10. European Commission 2008. Gender Equality Report. Framework Programme 6, EC, Brussels.

11. Alcon A, Peña T, Arrizabalaga P. Women physician and health research. Med Clin 2012;138:343-8.

12. Gupta N, Kemelgor C, Fuchs S, et al. Triple burden on women in science. A cross-cultural analysis. Curr Sci 2005;89:1382-6.

13. Ellemers N, Van Den Heuvel H, De Gilder D, et al. The underrepresentation of women in science. Differential commitment or the queen bee syndrome? Br J Soc Psychol 2004;43:3.

14. Zimmer A. 2003. Women in European Universities. Final Report 2000-2003 of the Research and Training Network. http://www. women-eu.de (accessed 11 Jan 2012).

15. Aleixandre Benavente R, González-Alcaide G, Alonso-Arroyo A, et al. Valoración de la paridad en la autoría de los artículos publicados en la Revista Enfermedades Infecciosas y Microbiología Clínica durante el quinquenio 2001-2005. Enferm Infecc Microbiol Clin 2007;25:619-26.

16. Sullivan LW, Mittman SL. The state of diversity in the health professions a century after Flexner. Acad Med 2010;85:246-53.

17. Dinauer LD, Ondeck KE. Gender and institutional affiliation as determinants of publishing in human communications research. Hum Commun Res 1999;25:548-68.

18. Creamer EG. Assessing Faculty Publication Productivity: Issues of Equity, ASHE-ERIC Higher Education Report 26(2), Graduate School of Education and Human Development, George Washington University, 1998.

19. Long JS. Measures of sex differences in scientific productivity. Soc Forces 1992;71:159-78.

20. Mathews AL, Andersen K. A gender gap in publishing?. Political Sci Polit 2001;34:143-7.

21. Schneider A. Why don't women publish as much as men? Some blame inequity in academe; others say quantity doesn't matter. The Chronicle of Higher Education 1998;11:A14. 